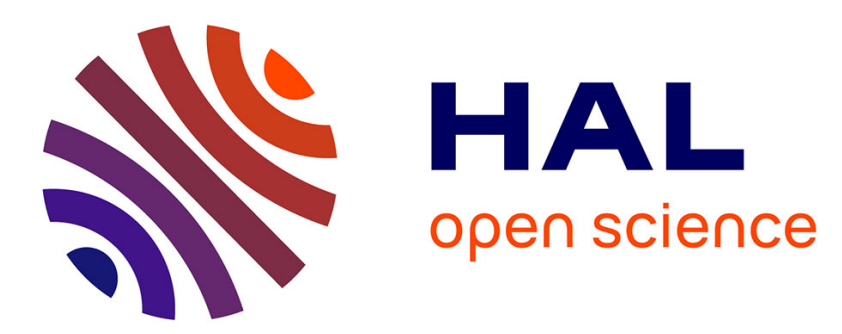

\title{
Modes de coordination instaurés par le pivot d'un réseau d'innovation : le cas d'un porteur de projet TPE Elodie Gardet
}

\section{To cite this version:}

Elodie Gardet. Modes de coordination instaurés par le pivot d'un réseau d'innovation : le cas d'un porteur de projet TPE. Revue management \& avenir, 2009, 26, pp.33-51. 10.3917/mav.026.0033 . hal-01291988

\section{HAL Id: hal-01291988 \\ https://hal.univ-grenoble-alpes.fr/hal-01291988}

Submitted on 24 Mar 2016

HAL is a multi-disciplinary open access archive for the deposit and dissemination of scientific research documents, whether they are published or not. The documents may come from teaching and research institutions in France or abroad, or from public or private research centers.
L'archive ouverte pluridisciplinaire $\mathbf{H A L}$, est destinée au dépôt et à la diffusion de documents scientifiques de niveau recherche, publiés ou non, émanant des établissements d'enseignement et de recherche français ou étrangers, des laboratoires publics ou privés. 


\title{
Modes de coordination instaurés par le pivot d'un réseau d'innovation : le cas d'un porteur de projet TPE
}

\author{
par Elodie Gardet
}

\section{Résumé}

Cet article a pour objectif de comprendre les modes de coordination qu'un porteur de projet TPE peut mettre en œuvre avec ses différents partenaires. Nous souhaitons montrer que les modes de coordination ne sont pas statiques mais qu'ils évoluent avec l'avancement du projet, d'où le choix d'une étude de cas menée sur 18 mois. Le réseau d'innovation a été observé de façon dynamique, au fur et à mesure de son déploiement afin d'étudier les éventuels changements.

\begin{abstract}
This article aims to understand the coordination modes that a SME bearer can implement with its different partners. We want to show that the coordination modes are not static but evolve with the project. That is why we have chosen a case study approach. The innovation network has been observed in a dynamic way to study the possible changes during its deployment.
\end{abstract}

Les modes de coordination, en réduisant l'incertitude et les comportements opportunistes, sont essentiels au sein de réseaux d'innovation (Dhanaraj et Parkhe, 2006). En effet, ces formes organisationnelles sont particulièrement propices à l'échange d'informations et à la transmission de savoir-faire, ce qui risque de favoriser les comportements opportunistes (Goerzen, 2007). En outre, le caractère souvent tacite du savoir et le faible degré de prévisibilité des résultats engendrent une incertitude élevée. L'enjeu de cette contribution est de comprendre les modes de coordination instaurés au sein d'un réseau d'innovation piloté par une TPE. En effet, rares sont les travaux empiriques se focalisant sur la compréhension du fonctionnement interne des réseaux d'innovation (Dhanaraj et Parkhe, 2006). La plupart des recherches sur les relations interorganisationnelles pour l'innovation analysent les raisons de la coopération, plutôt que son fonctionnement et adoptent une vision statique (à un moment $t$ de la coopération). Elles réalisent surtout des études cherchant à expliquer la performance des entreprises (Durand, Bruyaka et Mangematin, 2008 ; Gilsing et Nooteboom, 2006), dans des réseaux d'innovation pilotés par de grandes entreprises, et souvent dans les secteurs des biotechnologies et des 
nouvelles technologies. Pourtant, les PME et TPE innovent également et dans de nombreux secteurs. En 1999, on compte 1408 PME et TPE parmi les déposants de brevets par la voie nationale (dépôts publiés), soit $51 \%$ des personnes morales françaises déposantes $\left(\mathrm{OSEO}^{2}, 2004\right)$. Les activités de ces PME et TPE sont variées et les brevets déposés dans des secteurs tels que la fabrication de machines, l'industrie agroalimentaire, les biens de consommation, etc.

Cet article propose une approche dynamique des modes de coordination au sein d'un réseau d'innovation piloté par une TPE. Dans une première partie, nous expliquons les raisons nous ayant conduits à nous focaliser sur un réseau centralisé, piloté par un acteur unique à savoir le porteur de projet, puis nous réalisons une revue de littérature des principaux modes de coordination auxquels le pivot peut recourir. La deuxième partie présente l'étude monographique, conduite sur 12 mois afin d'étudier le rôle de l'avancement du projet sur les modes de coordination mis en oeuvre par le pivot. Enfin, l'analyse de l'évolution des modes de coordination au sein de ce réseau nous conduit à discuter le rôle de la phase d'avancement et de la dépendance du pivot sur l'évolution de ces modes.

\section{Modes de coordination instaurés par le pivot d'un réseau d'innovation}

Un réseau interorganisationnel peut être considéré comme une coopération entre au moins trois organisations juridiquement distinctes dans le but de réaliser un projet commun. (Lecocq, 2004). Nous nous focalisons sur les réseaux ayant comme projet commun de développer un projet d'innovation et montrons les spécificités de notre objet d'étude. Le réseau d'innovation, qui n'échappe pas au flou sémantique qui entoure le concept de réseau, appelle un effort de clarification et de différenciation par rapport à d'autres formes d'organisation. Dans la lignée des travaux d'Assens (2003), d'Inkpen et Tsang (2005) et de Dhanaraj et Parkhe (2006), nous définissons le réseau d'innovation comme un ensemble de relations avec des organisations diverses (publiques/privées ; partenaires/ prestataires), piloté par un pivot (le porteur de projet) dans le but de valoriser l'invention de ce dernier.

Si certaines recherches (Zajac et Olsen, 1993 ; Ring et Van de Ven, 1994 ; Das et Teng, 2002) ont relevé l'importance de la prise en compte de l'évolution des coopérations interorganisationnelles, rares sont celles (hormis celle de Reuer, Zollo et Singh, 2002) qui analysent l'évolution des modes de coordination. Notre recherche vise précisément à combler partiellement ce vide. Les recherches processuelles distinguent généralement trois stades qui ont le mérite d'être

2. Rapport sur les PME déposantes de brevets en France et leurs caractéristiques (comprenant le secteur d'activité) et leurs évolutions entre 1999 et 2004. 
spécifiques et communs à tous les projets d'innovation : stade 1, de l'invention au développement ; stade 2, du développement à la production ; stade 3 , de la production à la diffusion. Pour comprendre l'évolution des modes de coordination en fonction de ces trois stades, nous réalisons une synthèse de la littérature sur les principaux modes de coordination au sein de relations interorganisationnelles.

Les modes de coordination sont vus comme des arrangements entre unités économiques qui régissent les manières dont ces unités peuvent coopérer pour, ensemble, développer le projet d'innovation (Grandori et Soda, 1995). Cette définition a l'avantage de se focaliser sur les interactions au niveau stratégique - et non au niveau opérationnel (comme la répartition des tâches ou les moyens de communication). Les modes de coordination au sein de réseaux d'innovation étant multiples, nous proposons de retenir les principaux.

Degré de formalisme. La formalisation de l'échange permet d'indiquer les rôles de chacune des parties. Pour comprendre le degré de formalisation à l'œuvre au sein d'un réseau d'innovation, il est donc indispensable de définir ce que recouvre la formalisation de l'échange dans ce papier. Les modes formels, explicites et écrits, peuvent prendre plusieurs formes comme des procédures normalisées, des rapports techniques, un système de comptabilité analytique, des systèmes de budget/planification, des contrats et des accords de confidentialité (Das et Teng, 1998 ; Gulati, 1995 ; Uzzi, 1997). Les modes informels, implicites et verbaux regroupent la mise en place d'équipes communes (Grandori et Soda, 1995), de séminaires, réunions et transfert de personnel et des mécanismes pour la prise de décisions partagées.

Confiance. La confiance interorganisationnelle ${ }^{3}$ est définie comme une condition psychologique sous-jacente qui peut être la cause ou le résultat d'un comportement ou d'un choix (Mothe et Ingham, 2003, p.12). Les variations de risque et d'interdépendance peuvent altérer à la fois le niveau et la forme de la confiance. Elle n'est donc pas appréhendée comme un état permanent, mais plutôt comme un état temporaire qui évolue au fur et à mesure des interactions entre les membres du réseau d'innovation. En conséquence, au sein d'un réseau d'innovation, il semble exister un continuum entre méfiance à la confiance. La confiance a pour origine les termes latins fides (foi) et fidere (se fier à) et s'oppose à la méfiance (ne pas se fier) (Mothe et Ingham, 2003). Le cycle de la méfiance (mistrust cycle) débute lorsque les membres du réseau se concentrent sur leurs propres intérêts en niant le collectif (Butler, 1995). Certains membres ne concèdent qu'une partie minimum des informations à leurs partenaires, en pensant que ce comportement est réciproque. Dans cette recherche, la méfiance est considérée comme étant la « confiance trompée ».

3. Nous considérons, dans la lignée de Mesquita (2007) que la confiance interorganisationnelle a un statut propre. Pour une revue de la littérature sur la confiance, cf. Mesquita (2007), Mothe et Ingham (2003) ou Simon (2007). 
Répartition des résultats. Un des éléments essentiels de la coopération est la définition d'une règle de partage des résultats (Vassolo, Anand et Folta, 2004). La répartition dépend des normes de justice qui varient d'un groupe à l'autre, d'une culture à l'autre, etc. La répartition équitable des résultats est souvent perçue comme une incitation à l'effort pour les membres du projet et est censée favoriser la performance d'un projet d'innovation (Kabanoff, 1991). Al'inverse, la répartition égalitaire des résultats est entendue dans le sens d'uniformité, d'indifférenciation entre les membres du projet. Les membres du réseau d'innovation obtiennent une part équivalente des résultats, quelles que soient les ressources et compétences apportées au projet. Ce type de répartition s'avère risqué lorsque les membres contribuent au projet d'innovation dans des proportions différentes, le mépris des diversités créant des injustices pouvant altérer le bon déroulement du projet d'innovation.

Garanties. Les systèmes de garantie instituent une protection pour le lésé potentiel en rendant coûteuse la sortie du (ou des) membre(s) opportuniste(s). Nous proposons de distinguer les garanties immédiates, telles que les garanties financières ou les actifs spécifiques, des garanties différées (modes indirects, répercussions en $n+1$, telles que la réputation ou les futures opportunités d'affaires). Les modes directs permettent de maîtriser le comportement de membres dès leur sélection (répercussions en $n$ ) alors que les modes indirects reposent sur la capacité de porter atteinte à la réputation du membre, voire de l'exclure d'une future opportunité d'affaires (répercussions en $n+1$ ).

Résolution de conflits. Mohr et Spekman (1994) proposent six modes de résolution de conflits lors de relations bilatérales. Nous les adaptons et n'en retenons que cinq pour tenir compte des relations multilatérales au sein de réseaux d'innovation ${ }^{4}$ : la résolution conjointe du problème : les différentes parties s'engagent pour, ensemble, trouver une solution mutuelle au problème ; la persuasion : l'une des parties tente de persuader les autres membres qu'une solution donnée est la meilleure pour sortir de la situation de conflit ; la coercition : un (ou plusieurs) partenaire(s) contraint (contraignent) les autres à choisir la solution qu'il a retenue pour résoudre le conflit ; la sanction : le partenaire de la coopération est exclu ; faire appel à un tiers : celui-ci réalise un arbitrage entre les différentes parties prenantes (arbitre ou tribunal).

Le tableau 1 ci-dessous classifie les principaux modes de coordination proposés.

4. Mohr et Spekman (1994) distinguent la coercition de la domination, mais faute de définition précise, nous ne retiendrons pas cette distinction entre coercition et domination. 


\begin{tabular}{|c|c|c|}
\hline $\begin{array}{l}\text { Type de } \\
\text { coordination }\end{array}$ & Questionnement & Modes de coordination \\
\hline \multirow{2}{*}{$\begin{array}{l}\text { Régulation de } \\
\text { l'échange }\end{array}$} & \multirow{2}{*}{$\begin{array}{l}\text { Comment les membres se } \\
\text { coordonnent-ils dans un } \\
\text { réseau d'innovation? }\end{array}$} & $\begin{array}{l}\text { Degré de formalisme : existence ou non } \\
\text { de contrat et le nombre de clauses }\end{array}$ \\
\hline & & Confiance ou méfiance \\
\hline \multirow{3}{*}{$\begin{array}{l}\text { Dispositifs } \\
\text { d'incitation et } \\
\text { de sanction }\end{array}$} & \multirow{3}{*}{$\begin{array}{l}\text { Quels types de dispositifs } \\
\text { mobilisent-ils en cours de } \\
\text { transaction? }\end{array}$} & $\begin{array}{l}\text { Répartition des résultats : égalitaire ou } \\
\text { équitable }\end{array}$ \\
\hline & & Garanties : $\varnothing$, directes et/ou indirectes \\
\hline & & $\begin{array}{l}\text { Résolution de conflits : conjointe, } \\
\text { persuasion, coercition, sanction, appel à } \\
\text { un tiers (arbitre ou tribunal) }\end{array}$ \\
\hline
\end{tabular}

Tableau 1 : Coordination des membres du réseau d'innovation

Les principales recherches empiriques sur le fonctionnement interne d'une coopération se sont centrées, jusqu'alors, sur l'étude d'un ou deux modes de coordination à un instant $t$ (Poppo et Zenger, 2002 ; Mohr et Spekman, 1994 ; Mothe et Ingham, 2003). Trois stades du projet sont ainsi retenus : de l'invention au développement (stade 1), du développement à la production (stade 2), de la production au marché (stade 3). Par ailleurs, dans les relations d'échange entre les membres d'un réseau d'innovation, outre la phase d'avancement du projet, la dépendance du pivot apparaît comme étant une dimension importante pour comprendre les modes de coordination instaurés (Tinlot et Mothe, 2005). En effet, la dépendance n'est pas une caractéristique statique (Delerue et Simon, 2005). Elle peut évoluer au fur et à mesure de l'avancement du projet. Le réseau évolue au gré des coopérations et des ententes entre les différents membres (Larson, 1992). Selon les stades d'avancement du projet, les ressources et compétences indispensables à son déroulement changent. Le pouvoir de négociation de chaque membre est donc déterminé par les ressources qu'il apporte (Yan et Gray, 1994): physiques, financières, etc. ou les compétences et savoir-faire qu'il possède (réputation d'un partenaire, réseau de relations, etc.). Leur nature détermine le niveau de dépendance du pivot par rapport aux membres de son réseau, et donc son niveau de pouvoir (Blau, 1964).

\section{L'étude d'un réseau d'innovation piloté par une TPE}

Dans cette partie, nous étudions la mise en œuvre des modes de coordination à travers l'étude d'un projet d'innovation mené par un porteur de projet TPE. Nous présentons la méthodologie utilisée puis le réseau étudié.

\subsection{Méthodologie}

Afin de mieux comprendre l'évolution des modes de coordination au sein d'un réseau d'innovation, nous avons réalisé une étude de cas unique. Nous 
avons fait ce choix car notre questionnement est large et nécessite de suivre les événements dans le temps (Wacheux, 1996). Le réseau d'innovation choisi présentait un avantage certain quant à l'accessibilité des données et nous a permis d'étudier les relations entre le pivot et les membres sur plusieurs stades. Notre étude se base sur 9 entretiens semi-directifs, cinq journées d'observation passive ainsi que des données secondaires externes, comme les dossiers et articles parus dans la presse spécialisée et les sites Internet des membres (cf. tableau 2). La collecte des articles relatifs à Pinc\&pile fait suite à la lecture des Echos, de la Tribune et des "dossiers » ou des chroniques des Chambres de Métiers et d'OSEO.

\begin{tabular}{|c|c|c|c|c|c|c|}
\hline \multicolumn{5}{|c|}{ Données primaires } & \multicolumn{2}{|c|}{ Données secondaires } \\
\hline \multicolumn{4}{|c|}{ Entretiens } & \multirow{2}{*}{$\begin{array}{l}\text { Observation } \\
\text { passive }\end{array}$} & \multirow{2}{*}{$\begin{array}{l}\text { Données } \\
\text { internes }\end{array}$} & \multirow{2}{*}{$\begin{array}{l}\text { Données } \\
\text { externes }\end{array}$} \\
\hline \multicolumn{2}{|c|}{ Type de membre } & $\begin{array}{l}\text { Durée et } \\
\text { enregistrement }\end{array}$ & Date & & & \\
\hline \multirow{3}{*}{ Pivot } & \multirow{3}{*}{$\begin{array}{l}\text { Inventeur et } \\
\text { directrice }\end{array}$} & 2h19 (OUI) & $26 / 06 / 06$ & \multirow{9}{*}{5 jours } & \multirow{9}{*}{$\begin{array}{c}\text { Contrats (3) } \\
\text { Dossiers } \\
\text { concours } \\
\text { projet } \\
\text { innovant (1) } \\
\text { Courriels (15) } \\
\text { Courriers } \\
\text { avocats (2) }\end{array}$} & \multirow{9}{*}{$\begin{array}{c}21 \\
\text { articles } \\
\text { de } \\
\text { presse* }\end{array}$} \\
\hline & & 1h10 (OUI) & $09 / 11 / 06$ & & & \\
\hline & & 2h32 (OUI) & 08/03/07 & & & \\
\hline \multirow[b]{2}{*}{$\begin{array}{l}\text { Membres } \\
\text { juridiques }\end{array}$} & Cabinet d'avocats & $36 \min (\mathrm{NON} \#)$ & 20/11/06 & & & \\
\hline & $\begin{array}{l}\text { Cabinet en } \\
\text { propriété industrielle } \\
\text { prestataire }\end{array}$ & $13 \min (\mathrm{OUI})$ & 03/08/06 & & & \\
\hline $\begin{array}{l}\text { Membre } \\
\text { technique }\end{array}$ & Entreprise partenaire & $47 \min (\mathrm{OUI})$ & 06/07/06 & & & \\
\hline $\begin{array}{l}\text { Membre } \\
\text { financier }\end{array}$ & Partenaire public & 1h02 (NON) & 05/12/06 & & & \\
\hline $\begin{array}{l}\text { Membre } \\
\text { industriel }\end{array}$ & $\begin{array}{l}\text { Entreprise } \\
\text { prestataire }\end{array}$ & $40 \min (\mathrm{NON} \#)$ & $16 / 01 / 07$ & & & \\
\hline $\begin{array}{l}\text { Membre } \\
\text { commercial }\end{array}$ & Entreprise partenaire & $50 \min (\mathrm{NON} \#)$ & $14 / 11 / 06$ & & & \\
\hline \multicolumn{7}{|c|}{$\begin{array}{l}\text { Le \# symbolise les cas où les entretiens ne sont pas enregistrés du fait que ces membres entretiennent une } \\
\text { relation conflictuelle avec le pivot ou ont pour mission de défendre le pivot devant les tribunaux. }\end{array}$} \\
\hline
\end{tabular}

Tableau 2 : Synthèse des types de données recueillies

Les entretiens ont été réalisés auprès des différents membres du réseau d'innovation : le porteur de projet, les membres financiers, techniques, industriels, commerciaux et juridiques (organismes de conseil en propriété industrielle). Ces entretiens semi-directifs, d'une durée moyenne d'une heure ${ }^{5}$, cherchaient à appréhender l'évolution des modes de coordination au sein de Pinc\&pile et notamment ceux étudiés dans la littérature. Chaque entretien a fait l'objet de codages (Miles et Huberman, 2003), progressivement affinés au cours de la recherche. Nous avons également, lorsque cela était possible, recoupé

5. Toutefois, les entretiens réalisés avec le pivot, membre central du projet, étaient plus longs (en moyenne deux heures) que ceux réalisés avec les membres (en moyenne 41 minutes). 
les informations et effectué une triangulation entre les données primaires et secondaires. Notre recherche se focalisant sur la mise en œuvre de l'innovation en adoptant une approche dynamique, nous analysons le cas en fonction des trois principaux stades d'avancement d'un projet d'innovation : de l'invention au développement, du développement à la production et de la production à la diffusion.

\subsection{Présentation du cas Pinc\&pile}

Un constat a été à l'origine du projet de développement d'une pince à épiler jetable : en dépit de sa désinfection après chaque usage, l'hygiène de la pince à épiler n'est pas optimale. Par ailleurs, l'émergence de nouvelles attentes des clientes et les règles d'hygiène toujours plus draconiennes illustrent l'inadaptation des moyens utilisés par les esthéticiennes. Madame XB et son associée ont imaginé Pinc\&pile comme une réponse nouvelle à un problème pour lequel elles n'avaient, jusqu'alors, pas trouvé de solution viable, tant d'un point de vue technique (création du moule) que financière (coût de revient de la pièce trop élevé : $2 €$ par pince). Dans un premier temps (fin mars 2005), elles ont décidé de déposer une enveloppe Soleau, permettant d'assurer l'antériorité de l'idée. Puis en juin 2005, elles ont déposé un brevet avec une couverture mondiale : demande PCT (Patent Cooperation Treaty). L'architecture du réseau d'innovation évolue au gré de l'intensité des relations d'échange et des stades d'avancement du projet (cf. schéma 1). Les connexions entre les membres émergent ou disparaissent en fonction des stades d'avancement.

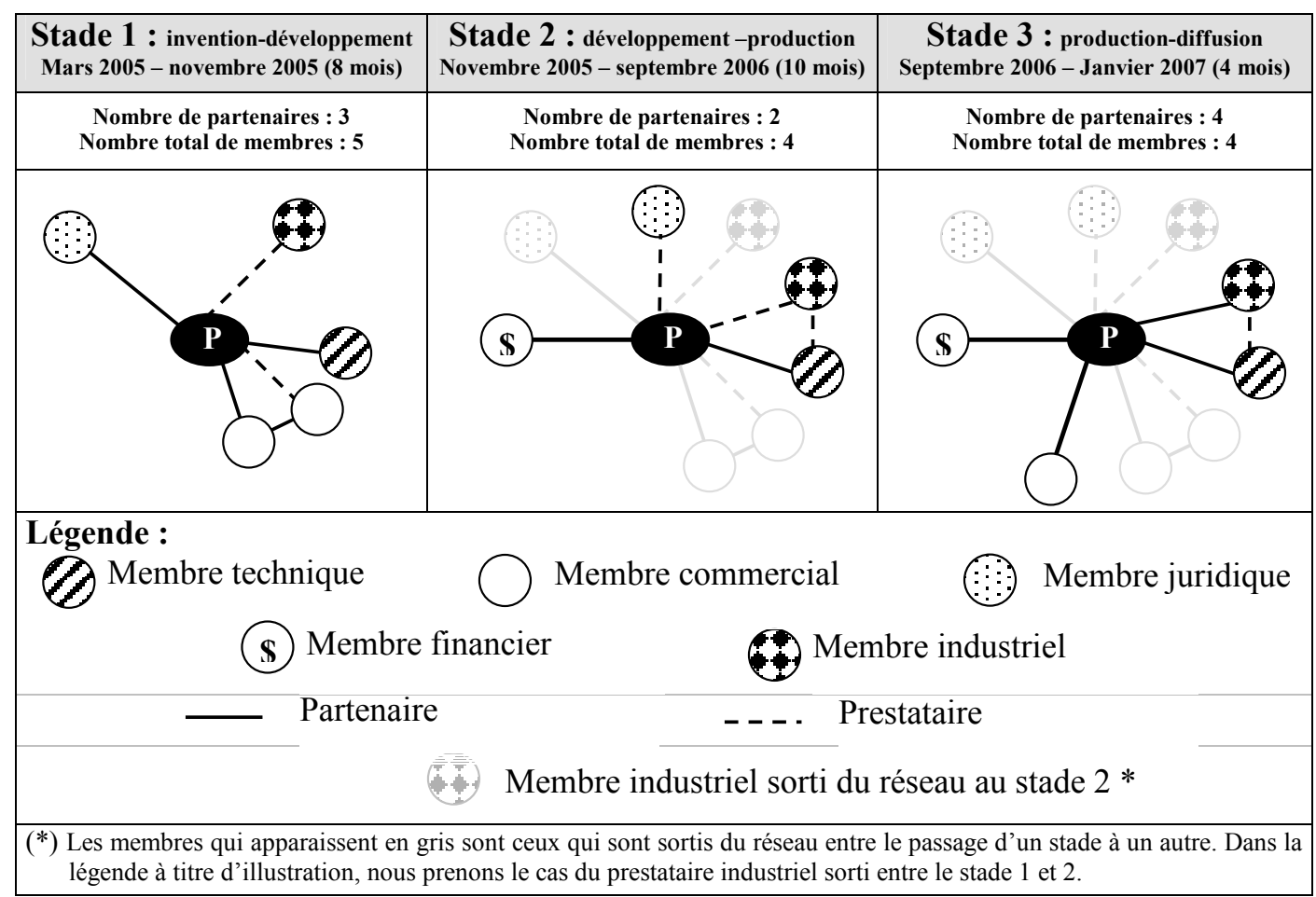

Schéma 1 : Structure du réseau Pinc\&pile au cours du projet 
Le projet Pinc\&pile nécessite un ensemble de ressources et compétences hétérogènes. Ainsi, pour développer son projet d'innovation, le porteur de Pinc\&pile a dû se coordonner avec de multiples organisations. Nous présentons dans une dernière partie les principaux modes de coordination mis en œuvre par le pivot au fur et à mesure de l'avancement du projet.

\section{Analyse des modes de coordination instaurés et discussion des résultats}

Afin de faciliter la lecture des résultats, nous présentons dans un premier temps les modalités mises en œuvre par le pivot pour chacun des cinq modes de coordination. Dans un second temps, nous présentons les jeux de coordination en montrant la nécessité de considérer non seulement le stade d'avancement du projet d'innovation mais également le degré de dépendance du pivot pour appréhender les modes de coordination instaurés.

\subsection{Principaux modes de coordination mis en œuvre par le porteur de Pinc\&pile}

Dans le cas Pinc\&pile, nous avons repéré les cinq modes de coordination identifiés dans la littérature et déterminé les modes utilisés selon les membres, ainsi que leur évolution.

La formalisation de l'échange. Tout en conservant un pouvoir de décision important sur les enjeux stratégiques du projet, le pivot a privilégié les relations informelles. II a demandé simplement à chaque membre de Pinc\&pile de signer un accord de confidentialité, ce qu'il a considéré comme l'une des premières formalités afin d'entamer la coopération (suite aux conseils fournis par son partenaire juridique au stade 1). Toutefois, ce document n'a pas toujours été signé dès les premiers échanges et le pivot ne lui a accordé aucune valeur de protection de son invention :

"Monsieur X (prestataire juridique, stade 2) m’a conseillé de faire signer un accord de confidentialité à chaque personne à qui je parle de mon projet. Je le fais pour lui faire plaisir, mais mon invention est brevetée et, si on doit me copier, ce n'est pas cet accord qui empêchera de le faire » (pivot, projet B, le 26/06/2006)

Durant le stade 1, hormis le contrat de confidentialité, le pivot n'a pris aucun engagement écrit, que ce soit avec le membre technique ou avec le membre juridique. Ils se sont mis d'accord sur les modalités du partenariat, au cours de réunions ou d'échanges téléphoniques.

A contrario, les membres industriels et commerciaux ont voulu contractualiser la relation. Le membre industriel a demandé $49 \%$ de quotes-parts des droits de propriété du pivot. Les deux membres commerciaux, eux, ont souhaité une 
licence exclusive d'exploitation. Après trois aller-retour (par fax) pour tenter de trouver un accord, le pivot, fatigué de cette " paperasse administrative " (pivot, projet $B$, le 26/06/2006), a opté pour la recherche de nouveaux membres, car à ce stade ils n'étaient pas indispensables au projet.

Au stade 2, la relation avec le membre financier public (la Chambre de Métiers) n'a pas donné lieu à la signature d'un contrat de coopération. En revanche, le pivot a dû rédiger un dossier similaire à un business plan (projet, faisabilité technique, étude de marché, bilan et compte de résultat prévisionnel). Avec le prestataire industriel, contacté par le partenaire technique, un contrat de soustraitance a été signé. Au cours du stade 3 , la relation d'échange avec le prestataire industriel s'est peu à peu transformée en partenariat, sans pour autant donner lieu à la signature d'un nouvel accord. Ces relations d'échange avec le partenaire technique puis industriel ont eu pour base un degré de confiance élevé.

La confiance. Le pivot de Pinc\&pile a coordonné l'intégralité des transactions. Toutefois, la grande majorité des tâches à haute technicité ont échappé volontairement à son contrôle (le pivot ne possédait pas les compétences techniques nécessaires à la réalisation du projet) et se sont déroulées par l'intermédiaire de son partenaire technique. Le pivot a donc accordé une confiance très élevée à son partenaire technique et lui a délégué une partie importante de son pouvoir :

Au stade 2, le nouveau prestataire industriel a été sollicité pour créer une nouvelle version du moule permettant de produire les pièces (car le moule proposé par le premier prestataire a abouti à la création de pièces difficiles d'utilisation), sous le contrôle du partenaire technique ayant déjà travaillé avec lui. Cette confiance interorganisationnelle s'est acquise progressivement, au fur et à mesure des relations d'échange. C'est dans le cadre d'interactions et du travail commun que la confiance mutuelle a pu naître ou disparaître. Avec le partenaire juridique (stade 1), une amie de longue date, une confiance ex ante existait entre les deux membres. Toutefois, la confiance n'est jamais totalement acquise. Dans cette relation, la confiance n'a pas permis de prévenir le conflit et elle s'est rapidement transformée en défiance :

" J'avais confiance avant qu'elle ne la trahisse. Elle était jalouse de mon idée et voulait petit à petit prendre ma place au sein du projet. Plus ça allait et plus notre relation se dégradait. Elle voulait tout gérer pour moi et ne me transmettait pas toutes les infos qu'elle obtenait sur le projet (...). Le jeu était devenu mesquin, nous avions chacune pour objectif d'obtenir plus d'infos que l'autre. Mais c'est un jeu sournois et pas vraiment bénéfique pour le projet » (pivot, projet B, le 08/03/07)

La répartition des résultats. Avec les prestataires, la répartition des résultats n'a pas entraîné d'intenses discussions (à l'exception du prestataire industriel du 
stade 1 qui a demandé $49 \%$ des parts du brevet déjà déposé). Les devis réalisés ont servi de contrat pour la rémunération. Le montant a été calculé en fonction des volumes horaires nécessaires à la réalisation de la commande et aux coûts engendrés.

Au cours du stade 1, le chiffre d'affaires devait être partagé de manière égalitaire entre le pivot et les partenaires technique et juridique. Cependant, suite au conflit avec son partenaire juridique, seul son partenaire technique, présent depuis le début du projet, touche $50 \%$ du chiffre d'affaires réalisé et peut communiquer sur le projet Pinc\&pile en tant que partenaire principal (« main partner »). Les deux membres retirent les mêmes bénéfices:

"Avec l'entreprise $X$ (le pivot) c'est un peu comme un mariage. On est uni pour le meilleur et pour le pire " (partenaire technique, projet B, le 06/07/2006)

Son prestataire industriel (stade 2), devenu partenaire (stade 3), ne bénéficie pas de la répartition égalitaire. Le pivot considère qu'il serait injuste qu'un membre devenu partenaire au stade 3 (là où les risques diminuent) profite " du même traitement » (pivot, projet B, le 26/06/2006) que son partenaire technique présent depuis la genèse du projet. La répartition des résultats se fait au prorata des engagements financiers fournis par ce partenaire industriel. II peut également utiliser l'image du projet pour communiquer auprès de ses clients sur ses compétences (comme, par exemple, la fabrication d'un seul moule pour fabriquer la pièce maîtresse de Pinc\&pile).

Les garanties contre les comportements opportunistes. Les modes de garantie ont souvent été unilatéraux et pris par les membres du projet et non par le pivot, ce qui s'explique par : sa taille (le pivot de Pinc\&pile était composé de deux personnes), sa faible capacité financière (le financement de ses investissements l'a contraint à dépasser sa capacité d'autofinancement), son inexpérience ne lui donnant pas la possibilité de bénéficier d'une forte crédibilité dans la conduite de projets innovants. Ces trois caractéristiques l'ont placé dans une situation de vulnérabilité par rapport aux membres potentiels :

" Je dois rapidement trouver des partenaires financiers car, l'année prochaine, je ne pense pas être en mesure d'entretenir mon brevet mondial. C'est d'ailleurs parce que j'en avais parlé à monsieur $X$ (partenaire commercial du stade 1) qu'il a voulu prendre des parts sur mon brevet. Comme, financièrement, je suis un peu juste, certains en profitent... » (pivot, projet $B$, le 26/06/2006)

Ces raisons expliquent qu'au stade 1, à l'exception du partenaire technique, les membres ont montré une certaine réticence à s'impliquer dans ce projet d'innovation. Certains (le prestataire industriel et les membres commerciaux du stade 1) ont profité de cette faiblesse pour réclamer des quotes-parts du brevet (garantie directe). Ces membres ont accepté de payer une partie des frais 
d'entretien de celui-ci (extensions à l'étranger, procédures diverses d'examens, etc.) à condition de bénéficier de quotes-parts. A contrario, les partenaires techniques (présents tout au long du projet) et juridique (stade 1) ne se sont pas garantis du risque d'opportunisme. La confiance réciproque acquise au cours de relations antérieures a été ici utilisée comme un substitut aux modes de garantie :

" J'ai déjà travaillé avec madame $X$, pour des demandes très différentes, mais je la connais suffisamment pour savoir que c'est une femme droite qui ne va pas me planter un couteau dans le dos. En plus, je ne vois pas bien comment...et quel intérêt elle aurait à faire ça " (pivot, projet B, le 03/08/06)

La résolution des conflits. Le pivot et les membres ont mis en place de modes de coordination préventifs (garanties, répartition des résultats ex ante) permettant de réduire la genèse des conflits. Cependant, ceux-ci n'ont pas été suffisants pour les éviter. Les conflits avec les membres commerciaux, les membres industriel et juridique ont été perçus par le pivot comme des menaces pour la pérennité du projet. Ceux-ci sont nés dès le début du projet. Le pivot s'est alors questionné sur son souhait de continuer le projet avec eux avant d'envisager la manière de résoudre le conflit :

" Je me suis demandé si je pouvais réussir sans eux. Après quelques nuits blanches et l'aide de monsieur $X$ (partenaire technique), nous sommes arrivés à la conclusion que personne n'est indispensable. Comme je savais que je ne voulais plus travailler avec eux, la discussion n'avait plus aucun sens " (pivot, projet B, le 26/06/2006)

Le conflit s'est soldé par la sortie des deux membres commerciaux, du membre industriel et juridique. Contrairement aux trois premiers, le partenaire juridique (stade 1) a refusé d'être évincé du projet et le pivot a dû recourir au tribunal. II a considéré que le pivot n'aurait jamais pu développer son invention sans sa présence et il a réclamé une compensation financière pour la perte de temps occasionnée. Le tribunal a tranché en faveur du pivot qui, même s'il a gagné le procès, a perdu quelques mois par rapport au planning prévisionnel de son projet.

A contrario, avec les deux principaux partenaires (technique et industriel), le conflit est considéré comme favorable à l'avancement du projet. Le pivot a perçu ces désaccords comme des sources de confrontation et de remise en cause de certains choix stratégiques ou techniques de Pinc\&pile et non comme une menace pour la suite du projet :

"Avec monsieur X (partenaire industriel) on n'est pas toujours d'accord sur le nombre de pièces à produire, mais c'est normal que nous ne soyons pas toujours d'accord sur tout. Cela montre qu'il s'intéresse vraiment à mon projet et c'est donc ensemble que nous essayons de trouver une solution à notre désaccord " (pivot, projet B, le 26/06/2006) 
Le pivot a tenté de canaliser le conflit en favorisant les discussions et en confrontant les points de vue. Les modes de résolution privilégiés ont été la résolution conjointe et la persuasion lorsque l'un des membres s'est considéré expert sur l'objet du conflit (par exemple, lors d'un désaccord sur la matière plastique à retenir, le partenaire technique tente de persuader les autres des avantages de telle ou telle matière). Dans cette situation, les discussions permettant de résorber le conflit ont été génératrices de progrès et ont favorisé l'avancement du projet Pinc\&pile.

\subsection{Mise en perspective des jeux de coordination}

Dans le réseau analysé, le pivot, malgré sa petite taille, n'est pas en situation de dépendance vis à vis de l'ensemble des membres. Ainsi, le degré de dépendance n'est pas global ni statique. II faut donc l'étudier catégorie de membres par catégorie de membres et au fur et à mesure de l'avancement du projet. Cette caractéristique différencie cette recherche de celles portant sur le degré de dépendance dans des alliances bilatérales (Delerue et Simon, 2005). Dans le réseau d'innovation étudié, le pivot peut, par exemple, être dépendant des membres financiers sans pour autant l'être des membres commerciaux. Que ce soit dans une relation bilatérale ou multilatérale, les sources de dépendance sont multiples : ressources, taille des partenaires, importance stratégique et urgence de la coopération (Tinlot et Mothe, 2005). Ainsi, au stade 1, le pivot a eu besoin, en priorité, de ressources juridiques afin de protéger son invention. Les membres commerciaux et industriels n'ont pas été indispensables. Le partenaire technique est apparu lui, comme essentiel car il assurait la faisabilité technique du projet. Au stade 2, le pivot a été dépendant de tous les membres à l'exception du prestataire juridique qui se chargeait uniquement de gérer le conflit avec le premier partenaire juridique. Cette dépendance est principalement née de la difficulté du pivot à trouver des membres pouvant les remplacer :

"Si mon partenaire technique m'avait lâchée en cours de route, je ne suis vraiment pas certaine que le projet aurait abouti. Je pense que des partenaires de cette qualité sont vraiment très très rares et j'aurais eu beaucoup de mal à le remplacer " (pivot, projet $B$, le 08/03/07)

Au stade 3 , le pivot a disposé de produits prêts à être commercialisés. II est alors plus difficile pour le partenaire de négliger le travail effectué conjointement avec le pivot. Dans ce cas, il y aurait rupture de leur relation de coopération aux dépens du partenaire qui ne bénéficierait que dans une moindre mesure des retombées de la réussite du projet.

Dans le tableau de synthèse du cas Pinc\&pile (cf. tableau 3, page suivante) sont mis en évidence les principaux modes de coordination instaurés selon le stade d'avancement et le degré de dépendance du pivot. Les modes de coordination ayant entraîné des retards quant au déroulement du projet Pinc\&pile sont 
soulignés. Afin de faciliter la compréhension de ce tableau, une lecture détaillée des six configurations et des jeux de coordination est proposée.

Configuration 1 (stade 1 - pas de dépendance) : le degré de confiance entre le pivot et les trois membres (un prestataire industriel et deux membres commerciaux) est plutôt faible car, dès leur entrée dans le réseau, ces membres ont fait pression sur le pivot pour imposer leurs conditions de coopération. Du fait de l'inexpérience et de la petite taille du pivot (confiance faible), ces membres ont non seulement souhaité une répartition des bénéfices au prorata de leurs apports, mais également des garanties directes très importantes : quote-part des droits de propriété intellectuelle et licence d'exclusivité (ces garanties n'ont finalement pas pu être prises, c'est pourquoi elles apparaissent en italique dans le tableau de synthèse). Comme le pivot n'est pas encore au stade de production (stade 2), il n'y a pas d'urgence de coopération. Le pivot n'est pas dépendant de ces trois membres, ce qui explique que la résolution des conflits a entraîné leur sortie. Ce choix du pivot s'explique par l'absence de dépendance du pivot vis-àvis du prestataire industriel et des deux membres commerciaux. Si ces membres avaient été indispensables au déroulement du projet, le pivot aurait agit d'une manière différente. De plus, la formalisation de l'échange est faible et aucun contrat écrit ne les engage les uns vis-à-vis des autres. 


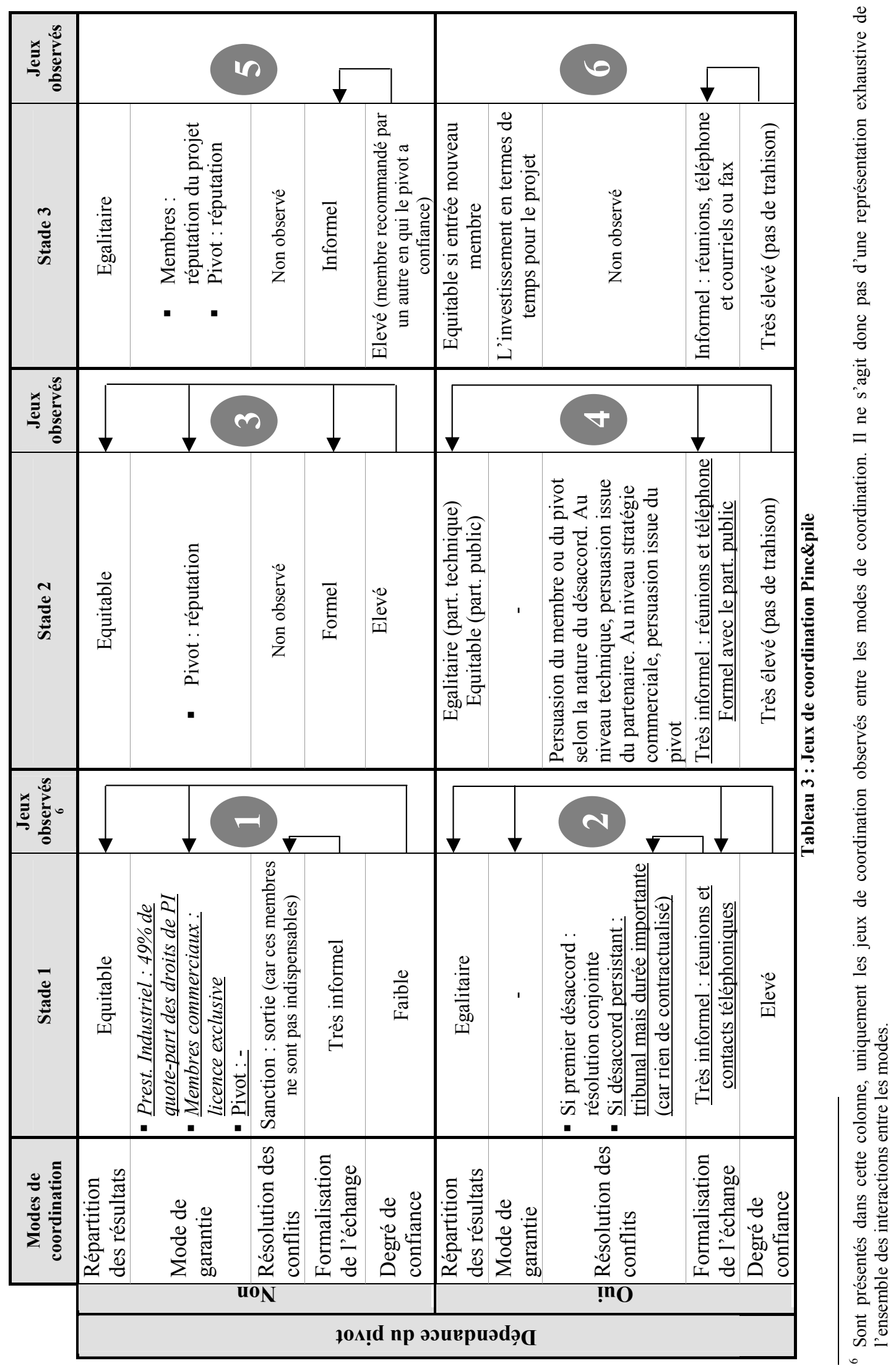


Configuration 2 (stade 1 - dépendance) : le pivot noue des relations peu formalisées avec ses partenaires juridique et technique (signature d'un accord de confidentialité), car il leur fait confiance (relations antérieures d'affaires). Les résultats prévisionnels sont répartis de manière égalitaire : chacun pense que l'autre fera son possible pour mener à bien le projet. Le pivot n'a pas pris de garanties contre les comportements opportunistes, ce qui devient problématique dès lors qu'émerge un conflit lié à la coopération. Avec ces deux partenaires, le pivot n'a pas prévu, en amont de leur relation, des modes permettant de faciliter la résolution de tels conflits (garanties, clauses contractuelles, etc.). Les relations informelles et la confiance sont les modes de coordination dominants. Dès lors que la confiance s'estompe, voire disparaît à cause de conflits (cas de la relation avec le partenaire juridique), le faible degré de formalisation rend l'arbitrage extérieur délicat, faute de preuve tangible. Dans le cas de Pinc\&pile, le tribunal de commerce a été sollicité. En faisant intervenir le tribunal et en excluant le membre juridique au stade 1 , le pivot a pris le risque de mettre en péril sa réputation au cours des stades suivants et, par conséquent, de rencontrer des difficultés afin de trouver un membre de substitution :

"Même si le tribunal nous a donné raison, c'est toujours délicat pour la suite. Les entreprises susceptibles d'intégrer le projet ne connaissent pas forcément bien l'histoire et peuvent s'arrêter au constat que nous avons rencontré de gros conflits avec telle entreprise, et se dire, si elle a eu des problèmes avec cette entreprise, pourquoi pas avec nous. C'est un échec de partenariat et ce n'est pas très vendeur... » (pivot, projet $B$, le 08/03/07)

Configuration 3 (stade 2 - pas de dépendance) : seuls le nouveau prestataire juridique et le prestataire industriel se trouvent dans cette configuration et les données recueillies apportent peu d'informations quant aux modes de coordination mis en œuvre. II s'agit d'une relation classique de prestation. Le prestataire met à disposition de son cocontractant (le pivot) son savoir-faire dans un domaine spécifique et déterminé. L'objet du contrat est un service que le prestataire propose à son client ("vente de service »). Les échecs dans ses relations antérieures incitent le pivot à davantage de méfiance (faible degré de confiance). Cela explique pourquoi, avec ces deux prestataires, il signe des contrats de prestation (fort degré de formalisation).

Configuration 4 (stade 2 - dépendance) : au stade 2, le pivot est dépendant du partenaire public financier et du partenaire technique. La relation est formalisée pour l'obtention de financements alors qu'elle est plus informelle lorsqu'il s'agit de mises au point techniques, l'échange se fait par l'intermédiaire de réunions et d'appels téléphoniques. Dans les deux cas, le pivot a confiance en ces membres car :

- avec le partenaire technique, il a des relations antérieures d'affaires satisfaisantes facilitant la confiance ; 
- avec le partenaire public, son image et ses objectifs (aider le pivot à accomplir son projet) lui confèrent une confiance innée.

Lors de légers désaccords sur la conduite du projet avec le partenaire technique, la persuasion du membre le plus compétent a permis de résoudre rapidement le problème (le pivot a une bonne connaissance du marché potentiel et persuade l'autre membre des voies de commercialisation à privilégier. A l'inverse, au niveau des choix technologiques, le membre technique impose son point de vue).

Configuration 5 (stade 3 - pas de dépendance) : le partenaire technique n'est désormais plus indispensable au projet, ayant fait la preuve de sa faisabilité technique au stade 2 . Comme la relation entre le pivot et le membre technique a été satisfaisante durant les deux précédents stades, les modes de coordination sont identiques à ceux observés au cours du stade 2 .

Configuration 6 (stade 3 - dépendance) : le prestataire industriel est devenu partenaire. Comme pour le partenaire technique, la confiance a été privilégiée ; en revanche, la répartition des résultats s'est faite de manière équitable afin d'éviter le sentiment d'injustice qu'aurait pu ressentir le partenaire technique présent depuis la genèse du projet. Les garanties ont été essentiellement liées au temps investi par les membres dans le projet.

Jeux de coordination observés. Pour chacune des six configurations, les flèches indiquent les principaux jeux de coordination observés (les liens entre les modes de coordination). Prenons les interactions observées au stade 1 (les jeux observés aux stades 2-3 sont semblables à ceux du stade 1):

- Dans la configuration 1, le pivot n'a pas signé de contrat avec les membres de son réseau. II lui a donc été relativement facile de les exclure du projet en ne leur confiant pas de nouvelle tâche ;

- Dans la configuration 2, il apparaît qu'une forte confiance interorganisationnelle a influencé le degré de formalisation (très informel), le type de répartition des résultats (égalitaire) et les garanties instaurées (inexistantes). De même, le degré de formalisation a eu des répercussions sur la manière de résoudre le conflit. Dans ce cas précis, il n'y avait aucun contrat signé donc pas de clause d'arbitrage. C'est pour cette raison que le tribunal a été saisi.

A travers l'étude du cas Pinc\&pile, nous montrons l'importance de considérer le stade d'avancement du projet et le degré de dépendance du pivot afin d'appréhender les modes de coordination mis en œuvre par le pivot. 


\section{Conclusion}

Cette recherche analyse les modes de coordination instaurés par un porteur de projet TPE pour développer son invention. Elle nous a permis de mieux comprendre le rôle joué par le stade d'avancement du projet et le degré de dépendance du pivot sur le fonctionnement du réseau d'innovation. De plus, par l'analyse du degré de formalisation, de la confiance, de la répartition des résultats, des garanties et de la résolution des conflits, nous avons mis en avant les possibles interactions entre ces cinq modes (appelées jeux de coordination), donnant ainsi une vision plus complète de la coordination au sein d'un réseau d'innovation centré.

Il est important de préciser que si cette recherche ne considère que le rôle du stade d'avancement et de la dépendance sur les modes de coordination, elle n'affirme en aucun cas que toute autre dimension n'a pas d'influence sur ces modes. Ce travail ne représente qu'une première étape vers une meilleure compréhension du fonctionnement des modes de coordination à l'œuvre au sein d'un réseau d'innovation. Ainsi, la première limite est relative à la non prise en compte du rôle potentiel de la personnalité du dirigeant de l'entreprise pivot dans l'instauration des modes de coordination (Géraudel, 2008). Or, la place primordiale du dirigeant dans le fonctionnement de la TPE laisse à penser que les modes de coordination peuvent être influencés par la personnalité de celuici. La deuxième limite concerne l'utilisation de modèles linéaires du processus d'innovation pour identifier les trois stades constituant un projet d'innovation. Cette modélisation présente l'avantage de caractériser le projet comme un processus comportant plusieurs stades. Pour chacun d'eux, nous avons analysé les modes de coordination mis en œuvre par le pivot. Mais cette approche est critiquable car trop stricte quant à l'ordre des stades. Une plus grande souplesse, considérant que certains stades peuvent notamment se chevaucher, permettrait une analyse plus réaliste. La troisième porte sur le fait que nous n'avons pas distingué le degré d'intensité de l'innovation : incrémentale ou radicale (Benner et Tushman, 2003), voire le type de compétences nécessaires à l'innovation : d'exploitation ou d'exploration (O'Reilly et Tushman, 2004). Compte tenu du degré d'incertitude, croissant avec le degré de nouveauté, il est probable que les modalités de coordination varient selon le type d'innovation. Enfin, la dernière limite concerne le phénomène étudié qui est très spécifique. En effet, cette recherche se focalise sur l'analyse de réseaux centralisés, composé d'organisations indépendantes et ayant pour objectif de développer l'invention protégée d'un porteur de projet. Toutefois, le cadre d'analyse (tableau 1), composé de cinq modes de coordination peut être transposé à d'autres contextes. Voici, quelques exemples de questionnements qui pourraient faire l'objet de futurs travaux : comment évoluent les modes de coordination si le réseau est piloté par un broker ? Qui met en place les modes de coordination si le réseau est composé de plusieurs chefs d'orchestre simultanément ? Qu'en est-il de la coordination au sein de 
coopérations souhaitant développer des innovations de procédé, ou de projet dans le domaine des services ? En effet, dans ces deux cas, les risques d'opportunisme sont potentiellement plus importants car l'invention est difficile à protéger (dépôt de brevets, dessins et modèles).

\section{Bibliographie}

P. Aghion, J. Tirole (1994), "The management of innovation », Quarterly Journal of Economics, Vol. 4, No. 109, p. 1185-1207.

C. Assens (2003), "Le réseau d'entreprises : vers une synthèse des connaissances », Management International, Vol. 24, No. 1, p. 120-136.

P. Blau (1964), Exchange and Power in Social Life, New York: Wiley.

D.L. Butler (1995), « Promoting strategic learning by post secondary students with learning disabilities », Journal of Learning Disabilities, Vol. 28, p. 170-190.

R.C. Calia, F.M. Guerrini, G.L. Moura (2007), « Innovation networks: From technological development to business model reconfiguration », Technovation, Vol. 27, No. 8, p. 426432.

J.B. Cullen, J.L. Johnson, T. Sakano (2000), « Success through commitment and trust: The soft side of strategic alliance formation », Journal of World Business, Vol. 35, p. 223241.

T.K. Das, B.S. Teng (1998), « Between trust and control: Developing confidence in partner cooperation in alliances ", Academy of Management Review, Vol. 23, No. 3, p. 491-512.

T.K. Das, B.S. Teng (2002), "The Dynamics of Alliance Conditions in the Alliance Development Process », Journal of Management Studies, No. 39, p.725-746.

$\mathrm{H}$. Delerue, E. Simon (2005), « Confiance, contrat et degré d'asymétrie dans les relations d'alliance ", Management International, Vol. 10, No. 1, p. 51-62.

C. Dhanaraj, A. Parkhe (2006), "Orchestrating innovation networks », Academy of Management Review, No. 31, Vol. 3, p. 659-662.

R. Durand, O. Bruyaka, V. Mangematin (2008), « Do science and money go together? The case of the French biotech industry », Strategic Management Journal, Vol. 29, No. 12, p. 1281-1299.

M. Fréchet (2007), « Les difficultés relationnelles dans les partenariats d'innovation : une approche par le contrat psychologique ", Finance Contrôle Stratégie, Vol. 10, No. 1, p. 125-148.

V.A. Gilsing, B. Nooteboom (2006), « Exploration and exploitation in innovation systems: The case of pharmaceutical biotechnology ", Research Policy, Vol. 35, No. 1, p. 1-23.

A. Goerzen (2007), «Alliance networks and firm performance: The impact of repeated partnerships », Strategic Management Journal, Vol. 28, No. 5, p. 487-509.

A. Grandori, G. Soda (1995), «Inter-firm networks: Antecedents, mechanisms and forms », Organization Studies, Vol. 16, No. 2, p. 183-214.

R. Gulati (1995), «Does familiarity breed trust ? The implications of repeated ties for contractual choice in alliances ", Academy of Management Journal, Vol. 38, No. 1, p. 85112.

V. Harmaakorpi, H. Melkas (2005), « Knowledge management in regional innovation networks: The case of Lahti, Finland », European Planning Studies, Vol. 13, No. 5, p. 641-59.

A.C. Inkpen, E.W. Tsang (2005), "Social capital, networks and knowledge transfer », Academy of Management Review, Vol. 30, No. 1, p. 146-165.

B. Kabanoff (1991), "Equity, equality, power and conflict », Academy of Management Review, Vol. 16, No. 2, p. 416-441.

A. Larson (1992), « Network dyads in entrepreneurial settings: A study of the governance 
exchange of relationships », Administrative Science Quarterly, Vol. 37, No. 1, p. 76-104. $X$. Lecocq (2004), « Une approche socio-cognitive de l'opportunisme : le cas d'un réseau interorganisationnel européen », M@n@gement, Vol. 7, No. 3, p. 109-135.

L.F. Mesquita (2007), « Starting over when the bickering never ends: Rebuilding aggregate trust among clustered firms through trust facilitators ", Academy of Management Review, Vol. 32, No. 1, p. 72-91.

M.B. Miles, M. Huberman (2003), Analyse des données qualitatives, 2e Edition, De Boeck.

J. Mohr, R. Spekman (1994), "Characteristics of partnership success: Partnership attributes, communication behavior, and conflict resolution techniques ", Strategic Management Journal, Vol. 15, No. 2, p. 135-152.

R. Morgan, S. Hunt (1994), "The commitment-trust theory of relationship marketing ", Journal of Marketing, Vol. 58, No. 3, p. 20-38.

C. Mothe, M. Ingham (2003), « La confiance au sein de coopérations : une étude de cas longitudinale », Management International, Vol. 7, No. 4, p. 17-31.

B. Nooteboom, H. Berger, N.G. Noorderhaven (1997), "Effects of trust and governance on relational risk », Academy Management Journal, Vol. 40, No. 2, p. 308-338.

OSEO (2004), Les PME déposantes de brevets en France : caractéristiques et evolutions, Hélène Perrin, Direction des Etudes et de la Stratégie, Etudes et bilans réalisés par les experts internes d'OSEO.

J. Pfeffer, G. Salancik (1978), The external control of organizations, Harper and Row Publishers, New York.

L. Poppo, T. Zenger (2002), « Do formal contracts and relational governance function as substitutes or complements? », Strategic Management Journal, Vol. 23, p. 707-727.

J.J. Reuer, M. Zollo, H. Singh (2002), " Post-formation dynamics in strategic alliances, Strategic Management Journal, Vol. 23, No. 2, p. 135-151.

P.S. Ring, A.H. Van de Ven (1994), " Developmental process of cooperative interorganizational relationships ", Academy of Management Review, Vol. 19, No. 1, p. 90-118.

P.H. Rubin (1990), Managing business transactions, controlling the cost of coordinating, communicating and decision making, The Free Press, New York.

E. Simon (2007), "La confiance dans tous ses états », Revue Française de Gestion, Vol. 33, No. 175 , p. $83-94$.

G. Tinlot, C. Mothe (2005), «Alliance asymétrique et pouvoir de négociation des partenaires : une relecture de l'alliance Matra-Renault », Management International, Vol. 10, No. 1, p. 31-49.

B. Uzzi (1997), « Social structures and competition in interfirm. networks: The paradox of embeddedness », Administrative Science Quarterly, Vol. 42, No. 1, p. 35-67.

R.S. Vassolo, J. Anand, T.B. Folta (2004), " Non-additivity in portfolios of exploration activities: A real options-based analysis of equity alliances in biotechnology », Strategic Management Journal, Vol. 25, No. 11, p. 1045-1061.

F. Wacheux (1996), Méthodes Qualitatives et Recherche en Gestion, Economica, Paris. W.P. Wu, W.L. Choi (2004), " Transaction cost, social capital and firms' synergy creation in chinese business networks: An integrative approach », Asia Pacific Journal of Management, Vol. 21, No. 3, p. 325-343.

A. Yan, B. Gray (1994), " Bargaining power, management control, and performance in United States-China joint ventures: A comparative case study », Academy of Management Journal, Vol. 37, p. 1478-1517.

E.J. Zajac, C.P. Olsen (1993), « From transaction cost to transaction value analysis: Implications for study of interorganizational strategies », Journal of Management Studies, Vol. 30, p. 131-145. 